


\title{
POR UNA CONEXIÓN DE LA RSE CON LA ÉTICA EMPRESARIAL
}

\author{
Autor: José Luis Fernández Fernández. ${ }^{1}$ \\ Cátedra de Ética Económica y Empresarial. \\ Universidad Pontificia Comillas.
}

\section{Resumen}

Tras constatar el hecho de la recurrencia de las crisis y los ciclos económicos y de explicitar las causas determinantes de la que venimos viviendo desde el año 2007, se lleva la reflexión hacia el concepto de RSE, tratando de revisar críticamente algunas de las aproximaciones más habituales desde el punto de vista teórico. La conexión con ciertas aproximaciones institucionales llevadas a efecto en los últimos tiempos, es aprovechada para apostar por un concepto de RSE alineado con una aproximación moral a la misma. Se aboga por un enfoque estratégico de la RSE que la conecte con la razón profunda de ser de la empresa, con su auténtica misión y fin objetivo. Esta perspectiva teleológica remite al marco que aporta la ética de la virtud, por entender que desde dicho enfoque es desde donde se podrá estar en condiciones de hacer que los valores morales de la RSE se consoliden en aras del

\footnotetext{
${ }^{1}$ jlfernández@cee.upcomillas.es
} 
Bien Común. Ello implica ir más allá de los requerimientos legales, sí; pero también más acá de la opción puramente voluntaria y, a las veces, arbitraria.

Palabras clave: Crisis Económica; Concepto de RSE; Ética Empresarial; Dimensión ética de la RSE.

For a Corporate Social Responsibility linked to Business Ethics.

\begin{abstract}
After assuming the fact of the recurrence of economic cycles and crises, and after identify some of the causes for the one we are living in nowadays, the article critically reflects on the concept of CSR from a theoretical point of view. In order to connect some recent institutional providence with a robust idea for CSR, it will be bet for a moral approach, where CSR-beyond the legal requirements; but far from any kind of arbitrary practices- is understood from the strategy and in line with the telos, i.e., the very raison d'être for companies and businesses in society, in search for the Common Good.
\end{abstract}

Key words: Economic crisis; CSR concept; Business Ethics; CSR's ethical dimension.

\title{
1. LAS CRISIS, LOS CICLOS Y LAS POLÍTICAS ECONÓMICAS.
}

En este artículo vamos a hablar de empresa y de Responsabilidad Social de la Empresa. Ese será nuestro problema de investigación. Y lo haremos desde un contexto de crisis sistémica que, prolongada ya desde hace años, ha afectado de manera dramática a muchas empresas y ha dado paso a un debate de cierto calado respecto a lo que la RSE pueda representar en circunstancias y coyunturas como las que se viven. La propuesta que mantendremos, al hilo de la concreta pregunta de investigación -¿qué relación hay entre RSE y la Ética Empresarial?-, será la de enfatizar la conveniencia de conectar la RSE con la Ética, como modo de hacer que aquella pueda ofrecer planteamientos más creíbles desde el punto de vista práctico y mejor sustentados desde la perspectiva teórica. Pero antes, digamos algo sobre los ciclos económicos, las crisis y las providencias que buscan su superación. 
Las crisis económicas son un fenómeno recurrente a lo largo de la entera historia de la humanidad (Comín Comín, 2011; Clough, 1990); y cabe decir que muchos de los motivos dinamizadores de la Economía, entendida como ciencia -sobre todo, a partir del siglo XIX- enlazan, sin duda, con la voluntad de comprender y explicar el ciclo económico en el conjunto (Ekelund, 2008) de sus cuatro fases: expansión, auge, desaceleración y recesión; a efectos de anticipar la ulterior dinámica de las recuperaciones, las alzas y las consiguientes épocas de prosperidad (Tortella, 2010).

En el siglo XX se generalizó el estudio científico de las crisis y los ciclos, con la aplicación sistemática del análisis de datos estadísticos y de series econométricas. Destacan de manera especial a este respecto el economista ruso Nikolai Kondratieff (Kondratieff, 1944) y el norteamericano Wesley C. Mitchell (Mitchell, 1927). Pues bien, todo lo que se sabe respecto a los ciclos, se ha venido gestando desde la voluntad práctica de identificar y poner en funcionamiento las palancas políticas más conveniente para anticipar las crisis y dulcificar los periodos de recesión económica. Esto fue particularmente cierto a lo largo de la década de los años treinta del pasado siglo, durante la Gran Depresión. Y como elementos que merecen ser anotados a este respecto, estarían, de una parte, las providencias empíricas y las aplicaciones prácticas emanadas del llamado New Deal, obra del presidente norteamericano del momento: Franklin Delano Roosevelt y de otra la obra teórica de John Maynard Keynes, de la que destaca el clásico libro, publicado en 1936, que lleva por título: Teoría general de la ocupación, el interés y el dinero (Keynes, 2004).

El papel que desde entonces se hubo de atribuir al Estado en la gestión de la dinámica económica de la sociedad, supuso un punto de inflexión definitivo a la hora de manejar a través de las políticas, las variables macroeconómicas. En todo caso, los años que van desde el final de la Segunda Guerra Mundial -digamos mejor, desde la firma en el año 1944 de los acuerdos de Bretton Woods- y la mitad de los años 70 -precisamente hasta que se desencadenara la gran crisis del petróleo a la que más arriba a mediados de los años 70, son los tiempos dorados del keynesianismo, de lo que se ha denominado el pacto keynesiano, esto es: una suerte de contrato social implícito entre el trabajo y el capital. Constituyen también el momento más emblemático de la denominada Pax americana, o de los otros califican como los Treinta Gloriosos; en suma: la edad de oro del fordismo (Dortier, 2011).

Se estaba en presencia de un capitalismo renovado, un capitalismo de concertación que, supuestamente habría acabado por aprender la lección de la gran crisis de la década de los años 30. La receta, parecía clara: reglamentación de la economía y de las políticas de redistribución; e intervencionismo estatal en la dirección de la dinámica económica y en el control directo de los servicios considerados estratégicos.

Ahora bien, nada, ni en Economía ni en cualquier otro aspecto de la vida, permanece inalterable por mucho tiempo. Y, en efecto, así aconteció también con el ciclo virtuoso de los treinta años de crecimiento ininterrumpido. En consecuencia, también el keynesianismo hubo de rendir etapa. Había llegado el tiempo favorable 
para los que volvían a confiar en el mercado libre como pieza indiscutible del desarrollo económico. Había que cambiar el paradigma. Y fue entonces cuando economistas como Ludwig von Mises (Mises, 1995; Mises, 2011), Friedrich Hayeck (Hayek, 2009)... y Milton Friedman (Friedman and Friedman, 1966; Friedman, 1967) hubieron de ver reconocidos sus enfoques liberales como respuesta adecuada a unas circunstancias nuevas y a una profunda crisis económica. Una más, otra de tantas; pero, como siempre, distinta a las anteriores -ahora se hablaba de estanflación- y para la que no había previstos mecanismos de antídoto desde el recetario keynesiano.

La nueva política -orientada por los postulados del conocido como Consenso de Washington- apostaba por nuevas políticas de oferta; esto es: por un mercado libre y competitivo; por el librecambismo; por la desregulación de los mercados; por las privatizaciones de las empresas estatales y de las agencias económicas cuya gestión había venido estando encomendada a los gobiernos; y sobre todo, por el abandono de las políticas fiscales y monetarias discrecionales que hasta entonces habían formado parte del modo generalizado de proceder por parte de los Estados (Comín Comín, 2011).

Hay quienes afirman que fue precisamente el triunfo de las políticas económicas neoliberales del último tercio del siglo XX y los primeros compases del XXI lo que, junto al colapso de los sistemas de economía planificada y el desarrollo de las tecnologías de la información y las comunicaciones, dio lugar a la globalización económica que hoy vivimos; y a la preeminencia de lo financiero -de lo que se ha dado en llamar capitalismo financiarizado (Lapavitsas, 2009) - que, en última instancia, hubo de resultar determinante a la hora de alimentar una burbuja hipotecaria que, al estallar, originó la actual crisis.

A partir de la década de los años ochenta del pasado siglo, tienen lugar importantes innovaciones financieras (Gómez-Bezares, 1995). Ahora bien, en paralelo a dichas innovaciones, se debe destacar también el dato cierto de que las burbujas bursátiles fueron también realidades recurrentes. De hecho, se multiplicaron a partir del año 1998, y afectaron a las distintas bolsas mundiales, ya sean las bolsas de valores, ya a las de materias primas, a las de alimentos, a las de combustibles o a la del mercado hipotecario, organizada en torno al precio de las viviendas.

Si a aquellas innovaciones financieras en complejísimos productos derivados, sumamos el impulso liberalizador, la consigna de suprimir reglamentos y regulaciones, tendremos un panorama bien delineado para mejor comprender la génesis, y el desarrollo de la crisis por la que aún atravesamos. Entre los estorbos que los nuevos tiempos financieros identificaban, rémora nada menos que del New Deal, estaba la denominada ley Glass-Steagall. Esta ley, que acabó siendo abolida en el año 1999 en aras de la supuestamente imprescindible mayor flexibilidad de la que se estaban viendo privados unos mercados financieros, de sobra capaces -se decía- de autorregularse con prudencia, llegado el caso, había sido promulgada en el año 1933 y establecía normas muy estrictas para el funcionamiento de los bancos. En concreto, fjaba con nitidez la separación entre los bancos comerciales 
y los bancos de inversión. Tratando de evitar una mezcla indeseable de estrategias, la ley Glass-Steagall buscaba evitar que los agentes financieros invirtieran en activos a largo plazo el dinero que recibían a corto.

La actual crisis económica empezó, pues, a gestarse en este caldo de cultivo. Sin embargo, tuvo unas causas inmediatas que es posible referir, aproximadamente, en los términos que emplearemos en los dos párrafos siguientes.

En el año 2001 hizo explosión la burbuja de Internet; y de la euforia, se pasó a la crisis y al desconcierto. Los gobiernos más directamente concernidos, trataron de reactivar los mercados y de inyectar entusiasmo y nueva energía en el motor económico. Así la Reserva Federal bajó el precio del dinero, en dos años, del 6,5 al 1\%. Con lo que dio paso a la revalorización de un mercado inmobiliario que, por entonces, empezaba a despegar. En diez años, de una parte, el precio de las viviendas estadounidense se habría de multiplicarpor dos. Y de otra, los tipos de interés vigentes en los mercados financieros internacionales habrían de ser excepcionalmente bajos, con la lógica consecuencia de que los márgenes de intermediación que los bancos lograban por el negocio ordinario -esto es, el de captar pasivo y financiar operaciones de crédito- eran cada vez más bajos. Con la vista puesta en obtener mayores beneficios, la banca norteamericana en general diseñó una estrategia que, en esencia, vino a consistir en aumentar el número de operaciones realizadas; y en conceder préstamos más arriesgados para poder cobrar inetereses más altos (Abadía, 2009).

Estos préstamos se instrumentaron a partir de las denominadas hipotecas subprime o hipotecas de alto riesgo de impago. La ingeniería financiera, por su parte, empezó a moverse con innovadora creatividad, a fin de poder endosar el riesgo a otros inversores. Así, para cubrir la contingencia de los posibles impagos, los bancos norteamericanos que habían empezado a conceder créditos arriesgados titulizaron la deuda en paquetes que se llamaban MBS -Mortgage Backed Securitiesesto es: obligaciones garantizadas por hipotecas -unas prime; otras, subprime-que fueron dando lugar a variaciones sobre el mismo tema de los derivados financieros. Aparecerían las CDO -Collateralized Debt Obligations- las CDS -Credit Default Swaps-, las Synthetic CDO. Con ello, se convirtió la deuda que los clientes tenían contraída con los prestamistas en otros instrumentos financieros que, a su vez, colocaron entre otros inversores -fondos, bancos y cajas de ahorro de todo el mundo.

Todo el entramado estaba basado en dos hipótesis: de un lado, que los que tenían una hipoteca irían haciendo frente a los pagos mensuales comprometidos; y de otro, que el precio de las viviendas que se compraban con aquellos préstamos, seguirían subiendo. Sin embargo, no habían de ser las circunstancias tan bonancibles. A principios de 2007, estalló la burbuja inmobiliaria en Norteamérica; y los precios de las viviendas norteamericanas se desplomaron, el mundo financiero se hundió y el sistema estuvo en grave riesgo de venirse completamente a pique. 


\section{UN VEREDICTO SOBRE LAS CAUSAS DE LA CRISIS ACTUAL.}

Se cuenta que, cuando la reina Isabel II de Inglaterra visitó la London School of Economics, en noviembre de 2008, desconcertada y perpleja -por un lado, tenía ante sí a brillantes profesores y reputados economistas; y por otro, empezaba a notarse en serio una situación económica pavorosa a escala global- planteó al ilustre claustro de aquella venerable institución la siguiente pregunta: “Pero dónde estaban ustedes?" (Petrini, 2010: 29). Desde entonces, la interpelación ha venido sonando en múltiples foros de muy diverso tipo institucional; y aunque los consensos al respecto no son fáciles -unos tienden a culpar a los gobiernos; otros, a los mercados desbocados- la literatura parece indicar que estamos ya en condiciones de captar las claves interpretativas por las que se interesaba con su pregunta, nada ingenua, $\mathrm{Su}$ Graciosa Majestad.

No son pocas, en efecto, las publicaciones a disposición del gran público (Abadía, 2009; EFC, 2012; Eagleton, 2011; Ekaizer, 2012; Krugman, 2012; Marx, 2011; Torres López, 2011; Tortella \& Núñez, 2010), donde se da cuenta de cómo hemos llegado a donde estamos, tanto a escala global cuanto europea (European Commission, 2009), como por lo que hace referencia al caso español (Ortega, 2012), donde, además de haberse roto aquella especie de consencs keynesiano que imperaba durante los Treinta Gloriosos, parece haberse esfumado también la cultura de la transición de la que habíamos venido viviendo desde fines de los años 70 del pasado siglo.

Con todo, si hay un documento que merece ser destacado a este respecto, ése no es otro que el Informe emanado de una comisión ad hoc, constituida en 2010 en los Estados Unidos a requerimiento de la Fraud Enforcement and Recovery Act, con un encargo concreto de preguntarse y responder a esta cuestión central: "How did it come to pass that in 2008 our nation was forced to choose between two stark and painful alternatives -either risk the total collapse of our financial system and economy or inject trillions of taxpayer dollars into the financial system and an array of companies, as millions of Americans still lost their jobs, their savings, and their homes?" (Commission, 2011).

La comisión, tras un concienzudo y sistemático proceso de indagación, hizo públicos sus resultados. El veredicto, desde entonces, es de sobra conocido y a estas alturas ya no procede insistir al respecto. Baste retener el dato de que, quizás la espoleta final que haya hecho posible que la situación de crisis se generalizara, no haya sido otra que el estallido de la denominada burbuja inmobiliaria en los Estados Unidos.

En todo caso, lo que sí merece la pena resaltar son las nueve conclusiones que el Informe expone de manera explícita; y que pueden quedar parafraseadas de la siguiente manera: 
1. La crisis financiera era evitable.

2. Ciertos fallos generalizados de supervisión y regulación resultaron ser devastadores para la estabilidad de los mercados financieros.

3. Otra causa de la crisis hay que atribuirla a graves deficiencias en el gobierno corporativo y en la gestión de riesgos por parte de importantes empresas.

4. Endeudamiento excesivo, inversiones de alto riesgo y falta de transparencia, llevaron al sistema financiero por el camino de la crisis.

5. El gobierno tampoco reaccionó bien; y con su inconsistente respuesta, no hizo sino añadir incertidumbre y avivar el pánico en los mercados.

6. También fueron causas de la crisis el relajamiento en los criterios para conceder préstamos hipotecarios; y la titulización posterior de los préstamos.

7. La existencia de derivados over-the-counter, fruto de la desregulación llevada a dicho efecto en 2000, debe ser anotada, a su vez, entre las causas de la crisis.

8. También las agencias de rating pueden ser culpadas, por las opiniones vertidas acerca de la calidad de ciertos productos y determinadas operaciones.

9. La falta de ética y la distorsión de los valores habría acabado llevándose por delante la confianza que la ciudadanía había depositado en la accountability -esto es: la capacidad de rendir cuentas con transparencia- de instituciones y profesionles de muy diverso tipo.

Resultaría simplista explicar esta crisis sistémica, aludiendo en exclusiva a falta de ética y a la avaricia. Tiene sentido lo que con fina ironía señala Boatright (Boatright, 2010), citando a Hanlon y a Douglas W. Hubbard. El primero decía que nunca se debiera atribuir a la malicia lo que puede explicarse fácilmente apelando a la estupidez humana. Hubbar, por su parte, apostillaba que nunca debiera atribuirse a la maldad o a la estupidez, algo que puede ser perfectamente explicado por referencia a la acción de individuos, moderadamente racionales, que se mueven en sistemas complejos de interacciones por determinado tipo de incentivos.

En todo caso, incluso reconociendo que hubo muchos que actuaron de manera injusta, imprudente y claramente irresponsable, una crisis de tal calado no debe ser atribuida en exclusiva a unos cuantos y únicos responsables de haber actuado mal. Naturalmente tampoco procede ni culpar en general a todo el mundo -aunque todos: incluso, a veces, el ciudadano corriente, también-; ni por supuesto, atribuir a todos la responsabilidad por igual: Hay quienes tienen mayores cargos de qué responder, en función del puesto que ocupaban, del rol que se les asigna en el concierto socioinstitucional (Jin et al., 2013); y de la libertad de maniobra de que gozaban y estaban legítimamente investidos (Graafland and van de Ven, 2011).

Así, los garantes del bien común -agencias reguladoras, entre otros-, deberían haber evitado movimientos imprudentes; e impedido que los mercados estuvieran 
fuera del más mínimo control. Quienes ejercían el gobierno corporativo en las grandes compañías, deberían también haber estado en condiciones de oponerse a una deriva insostenible para la que había ya indicios bastantes.

Más allá de todo ello, hay un dato doble que llama poderosamente la atención cuando se lee el Informe de la Comisión al que nos estamos refiriendo. Por un lado, está el hecho de que no se hace mención explícita al papel que las empresas hayan podido desempeñar en el marco de las apelaciones a la Responsabilidad Social Corporativa. Es llamativo, toda vez que años antes al estallido de la crisis, el discurso al respecto venía siendo moneda de uso habitual entre las compañías más innovadoras y avanzadas del mundo. Y por otro, está el dato de que tampoco se haga apelación alguna en todo el Informe al papel que las instituciones académicas -universidades, escuelas de negocios, centros de investigación en Economía- hayan podido tener en todo el iter de la crisis.

Estas lagunas han favorecido, sin duda, que se estén llevando a cabo trabajos de investigación, que tratan de aportar algo de luz al problema. Y no tanto, para abundar sobre el posible papel que a la academia le haya podido corresponder en la génesis de la crisis; sino, sobre todo, para sugerir ciertos cambios conceptuales en el diseño curricular de los estudios de management, tales que pudieran convertirse en los remedios de cara al futuro, contribuyendo, de paso, a legitimar el quehacer docente e investigador (Boyle, 2004; Muijen, 2004; Podolny, 2009).

Por nuestra parte, con este artículo -en línea con lo que hemos venido haciendo en lo súltimos meses en la Cátedra de Ética Económica y Emprsearial de la Universidad Pontificia Comillas (Benavides Delgado, 2013)- pretendemos poner el foco en lo que, desde el punto de vista teórico y conceptual, la RSE significa o puede llegar a significar a partir de las providencias institucionales y las reflexiones que se han venido haciendo en el contexto de la crisis; desde el convencimiento de que la actual coyuntura, no sólo ha de ser vista como una amenaza para el desarrollo de la RSE, sino también como una cierta oportunidad (Yelkikalan and Köse, 2012: 295) para apostar por una gestión más responsable y un modelo de empresa más sostenible (Kolk, 2010; Fernández and Souto, 2009; Manubens, 2009; Sustainability Asset Management, 2011).

\section{HACIA UN NUEVO ENFOQUE INSTITUCIONAL DE LA RSE.}

Como veremos más abajo, no son pocas las referencias a las que acudir en busca de reflexiones sobre la relación entre la actual crisis económica y la Responsabilidad Social de las Empresas. De una parte, tenemos los abordajes que llevan a efecto los medios de comunicación -como no podía ser de otra manera-desde un enfoque periodístico y divulgativo. Un ejemplo de ello, sería el informe extraordinario, redactado por periodistas del diario madrileño El País el domingo 24 de 
noviembre de 2013 (García Vega, 2013; Santiago, 2013; Simón, 2013; Blázquez, 2013; Muñoz, 2013; Barba, 2013; Tapiador, 2013). Estos análisis contribuyen a concienciar a la opinión pública respecto al asunto y consiguen poner sobre el tapete inquietantes cuestiones de un calado teórico más profundo de lo que parece. Algunas, por modo de ejemplo, podrían ser las siguientes: ¿Qué quiere decir Responsabilidad Social de la Empresa a los ojos de una ciudadanía que vive en carne propia desde hace años una crisis pavorosa y que observa impotente cómo el paro aumenta; el consumo al que tradicionalmente venía accediendo, se recorta; el crédito no fluye y la financiación se estanca; los rescates de instituciones -bancos y entidades financieras, sobre todo (Jin et al., 2013)-, a menudo directamente culpables de lo que está ocurriendo, se lleva a efecto sin discusión política mayor, a expensas de todos -socialización de las pérdidas-; la acción social de la empresas se resiente, mientras que las recetas de austeridad y los recortes presupuestarios de parte de los gobiernos y las administraciones públicas se generalizan, y las reformas se acometen sin remedio, resquebrajando aún más el Estado de Bienestar y unos derechos sociales que parecían indiscutibles conquistas de la sociedad hace tan sólo unos años; y, sobre todo, cuando las perspectivas de recuperación aún se perciben muy lejanas?

Aparte de los artículos de opinión y divulgativos, también encontramos nuevas aproximaciones al fenómeno de la RSE -ya sea en sus aspectos más generales y de puesta al día; ya en el énfasis en algunos puntos específicos- desde instancias políticas de amplia trascendencia. Tal podrían ser los casos, de una parte, de los Principios Rectores sobre Empresa y Derechos Humanos, auspiciados desde la ONU (United Nations Human Rights, 2011); y de otra, la comunicación de la Comisión Europea sobre la estrategia renovada de la UE para 2011-2014 sobre la RSE (Comisión Europea, 2011; Volosevici, 2013). Ambos documentos, por lo demás, están teniendo en estos momentos traslación al caso español.

De un lado, desde el Ministerio de Empleo y Seguridad Social se está elaborando lo que en su día será el Plan Estratégico español sobre RSE. Y ello se hace atendiendo a las indicaciones y directrices de la UE con vistas a fomentar la RSE tanto en las empresas, cuanto en las administraciones públicas. El pasado 9 de octubre de 2013, el ejecutivo remitió al Consejo Estatal de Responsabilidad Social de las Empresas (CERSE) una primera versión del borrador del plan. En dicho documento, se contemplaban ochenta medidas, agrupadas -sin un orden y sistema claro- en diez áreas: promoción, educación, marca España, recursos humanos, inversión socialmente responsable, I+D+i, proveedores y emprendimiento, consumo responsable, medio ambiente, y cooperación al desarrollo. En parecidos términos de aplicación práctica se pueden también encontrar indicaciones en un documento previo, aprobado por el CERSE el 3 de mayo de 2011 (CERSE, 2011) y que lleva por título: El papel de la RSE ante la crisis económica: su contribución a un nuevo modelo productivo, la competitividad y el desarrollo sostenible. 
De otra parte, como traslación al ámbito español de las indicaciones implícitas en los Principios Rectores sobre Empresa y Derechos Humanos y de la exigencia de parte de la Comisión Europea de que se elaboraran durante el año 2013 los preceptivos planes nacionales, cabe dejar dicho que la Oficina de Derechos Humanos del Ministerio de Asuntos Exteriores y de Cooperación está llevando a cabo lo que -de momento también, borrador en segunda versión- haya en su día de ser el Plan Nacional de Empresas y Derechos Humanos (Ministerio de Asuntos Exteriores y de Cooperación, 2013).

Antes de proseguir analizando el tenor de lo que las publicaciones académicas han venido últimamente ofreciendo como respuesta a la pregunta por el papel de la RSE en el contexto de la crisis, conviene detenerse un tanto en algunas de las claves teóricas que están detrás de los nuevos abordajes institucionales emanados desde la Unión Europea y desde el propio gobierno español.

El 25 de octubre del año 2011, la Comisión Europea dirigía, como señalábamos unos párrafos más arriba, una comunicación al parlamento europeo, al consejo, al comité económico y social europeo y al comité de las regiones, sobre la estrategia renovada de la UE para 2011-2014 sobre la responsabilidad social de las empresas. Y lo hacía, de una parte, en el marco de la Estrategia Europa 2020; y de otra, desde la constatación de que "la crisis económica y sus consecuencias sociales han dañado en cierta medida la confianza de los consumidores en las empresas" y han hecho que aquéllos se interesen más por el comportamiento social y ético de éstas (Comisión Europea, 2011: 5).

En el documento que nos ocupa, tras dar somera cuenta del impacto de la política europea en lo tocante a RSE desde la aparición del denominado Libro Verde en el año 2001 (Volosevici, 2013), se constata cómo hay indicadores de progreso que avalan el dato de que, sin duda, se han venido produciendo avances en el ámbito de la RSE: número de empresas europeas que han suscrito el Pacto Mundial; número de organizaciones que tienen sitios registrados en el marco del Sistema de Gestión y Auditoría Mediambientales; número de empresas que han suscrito acuerdo transnacionales con organizaciones de trabajadores europeas o mundiales; la adhesión a la Iniciativa de Cumplimiento Social de las Empresas, para mejorar las condiciones de trabajo en las cadenas de suministro; el número de empresas que publican informes de sostenibilidad de acuerdo al GRI. Se añade también al haber, el hecho de haber desarrollado a través de la Alianza Europea para la RSE herramientas prácticas sobre algunas cuestiones clave, tales como, por ejemplo, la igualdad de género, la mejora del diálogo con los inversores sobre el rendimiento no financiero de la empresa, o la gestión responsable de la cadena de suministro. Se identifican también retos pendientes, desde el punto y hora en que se constata que hay muchas empresas que aún no han integrado plenamente ni en sus políticas, ni en sus prácticas, ni, por supuesto, en su estrategia de base las preocupaciones sociales y medioambientales. Es más: la Comisión es consciente 
de que sólo en quince de los veintisiete Estados miembros de la Unión Europea se han desarrollado políticas nacionales para impulsar la RSE.

Desde estos prenotandos, es desde el que debe leerse la interpretación de la RSE actualizada que la comunicación supone y que define ex novo de manera simplificada: La RSE es "la responsabilidad de las empresas por su impacto en la sociedad", a fin de, por una parte, maximizar la creación de valor compartido para los propietarios/accionistas y para los demás grupos de interés; y de otra, identificar, prevenir y atenuar posibles consecuencias adversas. Las propuestas concretas -programa de acción para el período 2011-2014- y las indiciaciones prácticas que el documento ofrece a continuación -papel de los poderes públicos, el diálogo social, la mejora de la visibilidad y difución de buenas prácticas, etc.-, se alinean con aquella interpretación renovada del concepto de RSE; con el reconocimiento de la naturaleza pluridimensional de la misma; y con la necesaria interconexión entre la RSE y el gobierno corporativo (Volosevici, 2013).

\section{La aproximación a la RSE desde la acción social y el business case.}

Junto a las aproximaciones periodísticas a la relación entre RSE y crisis económica; al lado de las providencias emanadas desde las instancias institucionales -ONU, Unión Europea, Gobierno de España- a las que acabamos de hacer mención, hay que dejar anotado que también la academia, la producción científica publicada en revistas de impacto y de relevancia internacional, ha venido abordando la relación entre la RSE y la crisis (Alpaslan C. M., 2009; Alpaslan, Green and Mitroff, 2009; Thomé, 2009). Lo ha hecho con perspectivas dispares (Manubens, 2009 ) y desde ángulos diversos -resulta llamativo a este respecto el énfasis que se pone en el abordaje referido a la identidad corporativa (Bravo et al., 2012) y a la imagen de marca que las empresas ganan o dejan de ganar, como ventaja competitiva, en el marco de la crisis (Yelkikalan and Köse, 2012), así como en el modo de capitalizar a favor propio, mediante la estructuración de estrategias y políticas de RSE, una mejora en la reputación, ganada desde acciones corporativas directamente orientadas a satisfacer las expectativas de sus grupos de interés (Pérez-Ruiz y Rodríguez del Bosque, 2012; Pérez and Rodríguez del Bosque, 2013), porque, sin duda, por decirlo con las propias palabras de algún autor: "We can suggest that CSR activities are important for overcoming some social problems caused by the crisis" (Yelkikalan and Köse, 2012: 395).

Tampoco deja de ser significativo, alineado con la perspectiva anterior, el enfoque orientado a la métrica del fenómeno, a la voluntad de medir las acciones de RSE (Gjolberg, 2009; Giannarakis and Theotokas, 2011; Turker, 2009), en línea con trabajos anteriores a este respecto (Maignan, 2000; Igalens and Gond, 2005). En este sentido merece ser mencionado cómo hay quien encuentra correlaciones positivas entre la RSE en tiempos de crisis, entendida como prácticas activas de acción social para paliar los problemas derivados de la crisis 
-desempleo, fractura social-, y la buena reputación (Communication World, 2009). Ello explicaría el hecho de que, según unos autores (Giannarakis and Theotokas, 2011), sobre todo las grandes compañías, hayan mantenido -y a veces incrementado- el gasto en acción social entre los años 2007 y 2010. Otros, sin embargo (Giannarakis and Sariannidis, 2012), parecen venir a concluir, tras examinar el papel de la RSE en un entorno tan turbulento como el comprendido entre los años 2008 y 2010, que, de acuerdo al CSR Index, las empresas allí listadas, habrían sido menos socialmente responsables durante los años 2008 y 2009, para incrementar, posteriormente, su empeño a favor de la RSE en el año 2009-2010. Naturalmente, señalan los autores, "in order to strengthen their reputation and to get the license to operate" (Giannarakis and Sariannidis, 2012: 579). Hay que hacer notar que el CSR Index viene determinado por el Boston College Center y el Reputation Institute, para medir el desarrollo de la RSE de las 50 mayores empresas norteamericanas (http://www.bcccc.net/ index.cfm?pageId=2202). La metodología está basada en la determinación de las percepciones que el público tiene respecto a "three main dimensions: $\mathrm{Ci}$ tizenship. Governance and Workplace on a scale of 1 to 100; counting for over $40 \%$ of a company's reputation in the USA. Regarding the rest $60 \%$, reputation is constituted by four dimension, Leadership, Performance, Product/ Service and Innovation" (Giannarakis and Sariannidis, 2012: 582). Para dar idea de la relevancia que este tipo de herramientas de medición de impactos y de benchmarking tienen, debemos decir a renglón seguido que hay otros índices de similar factura en otros contextos y países. Puede consultarse en tal sentido, entre otros, el Corporate Responsibility Index (http://www.corporate-responsibility.com.au/), auspiciado desde Business in the Community y la Net Balance Foundation (http://www.netbalance.com/insights/).

Evidentemente, tras las métricas -incluso más allá de lo pragmático y aplicado que pueda resultar el benchmarking posible- late una comprensión determinada de la RSE, no siempre homologable con otras aproximaciones ni teóricas, ni de práctica de política económica. Como muestra de lo que se comenta, no deja de ser indicativo el controvertido caso de la India. En dicho país, la RSE viene entendida -de modo distinto a como ocurre en nuestro contexto español y europeo, al que más arriba hemos hecho referencia, donde la voluntariedad es rasgo incuestionable -como una obligación legal -artículo 135 de la reciente Companies Act 2013, que alguien calificó como: "a landmark initiative... first in the world...to involve the business in achieving national developing goals together with government and civil society": (http://articles.economictimes.indiatimes.com/201309-25/news/42394347_1_csr-spending-csr-index-new-companies-act)- de aportar recursos económicos por parte de ciertas empresas -concretamente, unas 8.000 a las que se les pide que contribuyan con el $2 \%$ de los beneficios- para atender a necesidades sociales durante el año fiscal 2014-2015. Cobra sentido, 
entonces, establecer listados y rankings de empresas, al modo como lo pretende llevar a efecto el India Institute of Corporate Affaires (http://www.iica.in/).

En línea con lo que acabamos de comentar respecto a la aproximación india al fenómeno de la RSE, cabe decir que la literatura académica ha venido discurriendo, al respecto de la RSE y la crisis, por el estudio del modo como han reaccionado las empresas - no ya los reguladores- en ámbitos geográficos distintos (Arévalo, 2010; Iamandi, et al. 2010; Adamu, 2009; Karaibrahimoglu, 2010; Naudé, 2009; Birth, 2008; Njoroge, 2009).

Más que entrar a valorar críticamente lo que tales aproximaciones significan, lo que sí acaba desprendiéndose con claridad a partir de ellas es, de una parte, el hecho de que sigue siendo difícil precisar con exactitud lo que se haya de entender últimamente por el concepto de Responsabilidad Social de la Empresa (Dahlsrud, 2008); y de otra, que esta crisis está siendo de hecho un verdadero test para las teorías sobre la RSE (Kemper and Martin, 2010). Respecto a lo primero, es interesante notar que, a pesar de la ingente literatura de los últimos años, aún no se ha podido llegar a un consenso, ni sobre la definición de la RSE (Benavides Delgado et al., 2012) -incluso, ni tan siquiera sobre las siglas que delineen el fenómeno -¿CSR?, ¿RSE?, ¿RS?...-; ni sobre los contenidos básicos de la misma; ni sobre los presupuestos teóricos que la animan. Nos encontramos a este respecto, como con agudeza se ha señalado (Zamagni, 2012), en una etapa de privatización de las definiciones, de la que se derivan graves incomprensiones y, muchas veces, polémicas estériles que en poco contribuyen a mejorar la gestión empresarial en aras del bien común. Por ello, entiendo que las propias teorías sobre la RSE debieran ser vueltas a considerar desde el marco que ofrecen las nuevas -y críticas- realidades que nos está tocando vivir en estos años. Al final de este trabajo habremos de retomar estas ideas.

En todo caso, se sigue la impresión de que, con más frecuencia de lo esperado, se viene a considerar que la RSE tiene que ver, sobre todo, con el gasto realizado por las empresas en programas y proyectos de acción social. Por consiguiente, en épocas como las presentes, de crisis estructural y persistente, la RSE habría de resentirse, como intuitivamente cabría pensar. Ello, como decimos, tal vez pueda ser cierto cuando nos referimos a la acción social de las PYME. Cosa distinta, a tenor con lo indicado supra, es lo que parece haber ocurrido con la acción social de las grandes compañías multinacionales.

Al margen de este debate, sobre el que no parece haber un consenso general, la tendencia observable a equiparar RSE con la acción social de la empresa, que ya en otro lugar revisamos de manera crítica (Andreu-Pinillos y Fernández-Fernández, 2011), tal vez sea debida al hecho de que muchas de las aproximaciones que se han venido haciendo al fenómeno -al menos desde los últimos treinta o cuarenta años-, sean tributarias de Carroll (Carroll, 1979); y sobre todo, de la conocida pirámide de RSC propuesta por dicho autor (Carroll, 1991; Carroll and 
Shabana, 2010), donde, expandiendo las categorías identificadas previamente por McGuire (McGuire, 1963); y con cierto paralelismo con lo que A. Maslow había indicado respecto a la dinámica de las necesidades y de la motivación humana (Maslow, 1975), identifica diversos estratos de responsabilidad por parte de las empresas: economic, legal, ethical and discretionary/philanthropic.

En primer lugar, vendría la responsabilidad propiamente económica, alineada, de una parte, con las expectativas que los accionistas tienen de obtener dividendos y plusvalías como resultado de una adecuada gestión empresarial de parte de los directivos de la empresa; y conectada de otra con la creación y el mantenimiento de puestos de trabajo, capaces de retribuir de manera adecuada a los empleados y trabajadores. Para ello, es preciso que la empresa esté atenta al mercado, que busque satisfacer las necesidades con buenos productos y servicios, que innove y progrese tecnológicamente. De no conseguirlo, de no ser capaz de cumplir con la dimensión económica de su actividad; esto es: si no es capaz de obtener beneficios, acabará haciéndola desaparecer de un mercado, inmisericorde con quienes no consiguen aportar valor suficiente. Esta suerte de primum vivere, cuando se lleva a cabo bien -como diría Friedman (Friedman, 1970), sin dolo ni engaño y dentro de la ley y los buenos usos mercantiles-, configura de facto, en nuestra opinión, si no la clave de bóveda, cuando menos, el cimiento firme de una RSE bien entendida y directamente emanada desde el propio corazón del negocio. Por simplificar, se podría decir que, con los considerandos pertinentes y a expensas de ulteriores precisiones respecto a la índole moral de la RSE -sobre todo en lo que respecta a consecuencias no intencionadas, pero colaterales a la actuación de la empresa (Zamagni, 2012)-, se podría decir que good business is good ethics... al menos, en principio.

Sabido es que Carroll añade a esta primera responsabilidad económica otras dimensiones de la RSE. La segunda dimensión es lo que tematiza bajo el rubro de las que denomina responsabilidades legales; que en el fondo vendría a ser una suerte de contrato social, firmado entre la empresa y la sociedad, en virtud del cual, aquélla se comprometería a buscar la consecución de sus objetivos económicos, respetando el marco regulatorio que la ley representa (Carroll, 1979). Ahora bien, más allá de lo legal, exigible de manera imperativa por las autoridades y las administraciones públicas, se ubica la tercera dimensión de la RSE, la de las responsabilidades éticas: Esta prolongación de los requerimientos legales cristaliza, entre otras cosas, en las expectativas que la sociedad maneja (Carroll and Buchholdtz, 2009) respecto al modo -ético, dialogante y respetuso- con el que le gustatría que la empresa se condujera en sus relaciones con todos grupos de interés y no sólo con sus dueños y accionistas (Freeman and Reed, 1983).

Como cúspide de las responsabilidades, sitúa Carroll la filantropía empresarial; es decir, aquella implicación voluntaria, por parte de la empresa -sin que la sociedad lo considere estrictamente obligatorio-, en la resolución de problemas 
sociales, más o menos directamente alineados con su núcleo central de negocio. Como es sabido, Michael Porter y Mark Kramer, prolongarán esta dimensión, llevándola hacia lo que denominan filantropía estratégica, como medio para conseguir una ventaja competitiva (Porter and Kramer, 2002; Porter and Kramer, 2006).

Es desde este paradigma de comprensión de la RSE -ampliamente compartido, sobre todo en el ámbito anglosajón- desde el que cobra sentido la interpretación que algunos hacen respecto al modo como las empresas han ido reaccionando ante la crisis. A modo de botón de muestra, cabe citar de nuevo a Yelkikalan y Köse, autores que articulan un discurso coherente con lo que se viene indicando, que la crisis tuvo diferentes efectos en los distintos niveles de la pirámide de la RSE postulada desde el modelo de Carroll. Lo afirman con las siguientes dos proposiciones: "The crisis poses a threat to the responsibility dimensions located at the base of the CSR pyramid" (Yelkikalan y Köse, 2012: 296); y "the crisis poses an opportunity for the responsibility dimension located at the top of the CSR pyramid" (Yelkikalan y Köse, 2012: 297).

Por nuestra parte, en este trabajo pretendemos abogar por una manera concreta de entender y conceptualizar la RSE. No será ni la única, ciertamente; ni tal vez la que vaya a ser mayoritariamente suscrita por todos los interesados en el asunto (Lawrence and Weber, 2011), sobre todo en lo que hace referencia a la evolución práctica de la misma: se habla de haber pasado de la CSR1, propia de los años 50 del siglo pasado, a la CSR4, identificable ya en la década de los 90 hasta el cambio de siglo (Frederick, 1986; Frederick,1994; Frederick, 1998). La manera que se defenderá aquí, no pretende en modo alguno ser contraria a lo que, grosso modo, pasa por representar el leit-motiv más recurrente y al que, por sintetizar, cabría referirse como el argumento a favor de la RSE por razones de inmediata rentabilidad. Esto es, por alguna de las múltiples y proteicas versiones del conocido como business case en favor de la RSE (Carroll and Shabana, 2010).

Bajo dicho concepto -Business Case for CSR- ha de entenderse toda línea argumental que, buscando convencer a las empresas de la conveniencia de implicarse decididamente a favor de la RSE, trata de hacer notar los beneficios económicos y de otro tipo -más o menos tangibles y cuantificables- que de dicha opción se pueden derivar para la empresa. Entre éstos, suelen citarse los siguientes: reducción de costes y minimización de riesgos (Kurucz et al., 2008; Smith, 2005); obtención de ventajas competitivas (Burke and Logsdon, 1996; Porter an Kramer, 2002; Bruch and Walter, 2005; Porter and Kramer, 2006; Privato et al., 2008); mejora de la imagen y la reputación (Fombrum and Shanley, 1990), que se traduce en un incremento de la legitimidad otorgada por la sociedad a la empresa (Suchman, 1995); obtención de situaciones gana-gana (Drucker, 1984), mediante creación de valor a través de sinergias (Kurucz et al., 2008; Wheeler et al., 2003). 
Como indica Carroll, cuando se examina la historia del fenómeno y de la conceptualización teórica de la RSE, se constata cómo la propia idea del business case ha ido evolucionando desde el principio, ya poniendo el énfasis en evitar externalidades negativas -lo que en términos éticos se conoce como el principio de no-maleficencia, de inmejorable pedigrí, toda vez que viene emparentado con el hipocrático primum non nocere-; ya apostando abiertamente por implicarse positivamente, buscando hacer el bien, desde la conocida variación utilitarista del famoso enlightened self-interest del mundo anglosajón, para el cual, good ethics is good business; o dicho de otra manera: que la ética es rentable (Zamagni, 2012).

Con todo, los argumentos que se orientan en esta dirección, también tienen sus limitaciones. Así, por ejemplo, se ha indicado que los consumidores pudieran no ser capaces de apoyar como se esperaría a determinadas empresas que apuestan decididamente por la RSE, debido al escaso poder que, con frecuencia, aquéllos pueden ejercer en el mercado. Por ello, se sugiere la conveniencia de que las administraciones públicas faciliten la decisión de compra a los consumidores que busquen apoyar las causas de RSE, mediante el desarrollo y la introducción de una etiqueta de RSE (Valor, 2008: 323). Desde otro punto de vista, se señala también como limitación al enfoque del business case, el hecho de que la asunción implícita entre una correlación positiva entre unas bien articuladas iniciativas de RSE y los resultados financieros de la empresa no siempre se mantienen o, en todo caso, no duran eternamente. Mintzberg, a este respecto, señala que más allá de un cierto nivel, no se produce retorno: "The stock market is willing to reward social responsibility only to a point. It pays to be good but not too good" (Mintzberg, 1983). Y, abundando en las limitaciones del enfoque,Williamson et al. (2006) afirman que, dado que el entorno en el que operan las PYME no suele ser proclive a compensar las prácticas de RSE, habría que incentivar que dichas prácticas se lleva a efecto, moviendo la palanca regulatoria y no fiando tanto en un interés empresarial que no siempre se ve recompensado adecuadamente por parte del mercado.

\section{TAXONOMÍAS Y PERSPECTIVAS TEÓRICAS PARA LA RSE.}

Parecen haber quedado ya un tanto aparcados debates antiguos sobre la pertinencia o la indeseabilidad de que las empresas tomen en consideración aspectos que vayan más allá de lo económico y decidan involucrarse en asuntos relativos a la RSE. Las posturas críticas a este respecto, son conocidas. Por simplificarlas, cabría decir que, en el fondo, lo que se vendría a afirmar sería que la RSE constituye una verdadera amenaza no sólo para el sistema económico capitalista, sino que supone un impulso suicida, capaz de hacer peligrar el régimen democrático de las sociedades libres en su conjunto (Friedman y Friedman, 1966; Friedman, 1970). 
El libre mercado es mecanismo suficiente, según esta aproximación, para garantizar la cooperación voluntaria que legitima a la empresa como agente económico creador de riqueza. Las cosas, sin embargo, no son tan sencillas -al menos en los mercados reales- y la supuesta mejora automática, tipo mano invisible, no pasa de ser una quimera (Zamagni, 2012). Por ello, es comprensible que, partiendo de la conveniencia y la necesidad de que la empresa vaya más allá de lo estrictamente económico, se haya producido el despliegue teórico del que hemos sido testigos en los últimos años; y que, a veces, ofrece un aspecto complejo y enmarañado.

No es de extrañar, entonces, que vengan apareciendo desde hace años -incluso se diría que últimamente proliferan-, apelando a distintos modelos de comprensión, artículos científicos que buscan poner cierto sistema y orden respecto a lo que se haya de entender por RSE tanto en la teoría de empresa, cuanto en la práctica empresarial (Klonoski, 1991; Carroll, 1999; Melé, 2007; Melé, 2008; Lee, 2008; Kraus and Brtitzelmaier, 2012).

Entre las taxonomías más habituales, cabría mencionar las siguientes -cada una con sus intuiciones bien orientadas y sus flancos más desguarnecidos; y con sus adalides y partidarios más o menos fervientes y convencidos: La teoría de la actuación social de la empresa o Corporate Social Performance (Bowen, 1953; Ackerman, 1973; Sethi, 1975; Wartick and Cochran, 1985; Wood, 1991); la teoría del accionista o Shareholder Theory (Friedman, 1966; Friedman, 1970; Jensen and Meckling, 1976; McWilliams and Siegel, 2001; Porter and Kramer, 2002; Porter y Kramer, 2006); el enfoque de la ciudadanía corporativa o Corporate Citizenship (Waddock and Smith, 2000; Wood and Logsdon, 2002; Matten and Crane, 2005); o la conocida teoría de los grupos de interés o Stakeholder Theory (Freeman and Reed, 1983; Freeman, 1994; Mitchel, Agle and Wood, 1997; Freeman and Philips, 2002).

Naturalmente, no ha sido la anterior la única forma de categorizar de manera sistemática por referencia a una tipología definida, los distintos abordajes teóricos y prácticos de la RSE. Así, por ejemplo, procede hacer mención del enfoque llevado a cabo por parte de Windsor (Windsor, 2006), quien identifica en el proceso evolutivo de la literatura referida a la RSE tres tipos distintos de teorías. El primer bloque de teorías, según este autor, estaría constituido por las que apuestan por enfatizar la concepción moral del fenómeno, buscando beneficiar al conjunto de los Stakeholders mediante el altruismo. Un segundo grupo de teorías -en línea con lo que, de alguna forma, señalaremos nosotros un poco más abajo- subraya los aspectos económicos, indicando que no habría por qué llevar a cabo acciones de RSE que supusieran un gasto innecesario; desde el entendimiento de que la creación de riqueza es ya, per se, el modo más genuino de contribuir desde la empresa al bien común. El tercer grupo, cercano a la concepción de la ciudadanía corporativa, aunque trata de articular lo ético con lo económico, según Windsor, no acaba de constituir propiamente un postura teórica de síntesis. 
Otra aproximación sugerente a estos aspectos taxonómicos es la que ofrecen Elisabeth Garriga y Domènec Melé en un bien trabajado artículo de hace unos años, que aspiraba "a delinear el territorio de las teorías sobre la RSE" (Garriga and Melé, 2004). Por supuesto, lo que en este artículo se ofrece está plenamente alineado con otros trabajos de similar factura a los que ya hemos hecho mención más arriba (Melé, 2007; Melé, 2008). Según Garriga y Melé, las teorías de la RSE podrían quedar categorizadas de acuerdo a los siguientes parámetros: 1 . Teorías instrumentales; 2. Teorías políticas; 3. Teorías integradoras; y 4. Teorías éticas.

Las Teorías instrumentales subrayarían el hecho -innegable, por lo demás- de que la empresa sea un mecanismo institucional orientado a la creación de riqueza y que, en consecuencia, la actividad social empresarial debiera ser coherente y estar en sintonía con la creación de valor para los dueños o accionistas -Shareholder Value Approach. Las Teorías políticas enfatizarían el poder social que las empresas tienen y abundarían en el modo de ejercerlo responsablemnete, cooperando con la sociedad, al modo como lo haría un buen ciudadano -Corporate Citizenship Approach. Por su lado, las llamadas Teorías integradoras, vendrían a decir que las empresas deberían hacer propias las demandas sociales -integrarlas en sus estrategias y políticas-, en tanto en cuanto, la existencia y expansión de la empresa, depende de no dejar sistemáticamente desatendidos aquellos requerimientos Stakeholder Approach. Finalmente, las Teorías éticas asumirían el hecho de que las relaciones entre la empresa y la sociedad se fundamentan en valores éticos y que las empresas debieran aceptar las responsabilidades sociales, por encima de cualquier otra consideración de tipo alguno, como una obligación moral. Desde esta perspectiva sería desde la que se estaría apostando de veras por un auténtico desarrollo sostenible, desde una teoría del Stakeholder, no sólo estratégica, sino también moral y normativa.

\section{PROPUESTA CONCLUSIVA.}

Sin desdeñar la pertinencia del hilo argumental ínsito en el discurso cercano a quienes consideran que la razón para apostar por la RSE haya de buscarse en el retorno económico esperable, por nuestra parte, consideramos que -sobre ser más que cuestionable, al menos la versión más burda y automática; pues la afirmación no se compadece siempre con la realidad de las cosas- se pueden ensayar otras aproximaciones teóricas en pro de la RSE -máxime en tiempos de crisis- en línea con algunas de las intuiciones a las que hacíamos referencia supra, cuando nos referíamos a las distintas aproximaciones y enfoques conceptuales de la RSE: Corporate Social Performance, Shareholder Theory, Corporate Citizenship y Stakeholder Theory.

Reconociendo los aspectos sólidos y también las limitaciones de cada una de las anteriores perspectivas teóricas, quisiéramos cerrar las consideraciones que 
hemos venido llevando a efecto a lo largo de este artículo, dedicado a reflexionar sobre el concepto de RSE en el marco de la crisis económica, apostando por una aproximación moral a la RSE; por una concepción estratégica (Napal, 2013) que conecte de veras la RSE con la razón profunda de ser de la empresa (Kelly and White, 2009); con su auténtica misión y fin objetivo; con el verdadero telos (Canals, 2010) y la dimensión ética de la actividad empresarial (Suárez Serrano, 2013; Natale, 2010). Y todo ello, desde el marco que aporta la ética de la virtud (Athanassoulis, 2013), por entender que, precisamente desde este enfoque es desde donde se podrá estar en condiciones de hacer que -más allá de los requerimientos legales, sí; pero también más acá de la opción puramente voluntaria y, a la veces, arbitraria- los valores morales de la RSE (Camacho Laraña et al., 2013) se consoliden (Zamagni, 2012) en aras del bien común.

La actual efervescencia institucional en materia de RSE a la que hemos hecho alusión detenida más arriba -ONU, Unión Europea, Gobierno de España- constituye una favorable ocasión para estimular el debate y, sobre todo, para apostar por un enfoque como el que aquí se propone de vincular la RSE con los valores éticos y la dimensión moral de la empresa y la gestión.

\section{BIBLIOGRAFÍA.}

ABADÍA, L. (2009), La crisis Ninja y otros misterios de la economía actual, Espasa, Madrid.

ACKERMAN, R. (1973), "How companies respond to social demands", Harvard Business Review, vol 51, núm.4, pp. 88-98

ADAMU, A. (2009), The Effects of Global Financial Crisis on Nigerian Economy. Recuperado el 4 de Diciembre de 2013, de http://ssrn.com/abstract=1397232 o http://dx.doi.org/10.2139/ssrn.1397232; http://ssrn.com/ abstract $=1397232$ o, http://dx.doi.org/10.2139/ssrn.1397232

ALPASLAN, C. M. (2009a), "Corporate Governance in the Context of Crises: Towards a Stakeholder Theory of Crisis Management", Journal of Contingencies and Crisis Management, pp.38-49.

(2009b), "Ethical management of Crises. Shareholder Value Maximisation or Stakeholder Loss Minimisation?", Journal of Corporate Citizenship, vol.36, pp.41-50.

ANDREU-PINILLOS, A. y FERNÁNDEZ-FERNÁNDEZ, J. (2011), "De la RSC a la sostenibilidad corporativa: una evolución necesaria para la creación de valor", Havard-Deusto Business Review, núm. 207 , pp. 4-21.

ARÉVALO, J. y otros (2010), "The impact of the crisis on corporate responsibility: the case of UN global compact participants in the USA", Corporate Governance, pp. 406-420. 
ATHANASSOULIS, N. (2013), Virtue Ethics, Bloomsbury, Chennai, India. BARBA, B. (2013), "Aquí hay dinero. La inversión en compañías socialmente responsables ofrece a las empresas mejor rentabilidad y financiación má económica", El País, Extra. Responsabilidad Social Corporativa. En el ADN de la empresa, pp. 12.

BENAVIDES DELGADO, J. y otros (2012), Glosario sobre Responsabilidad Social para la investigación y el debate terminológico, Universidad Pontificia Comillas, Madrid.

BENAVIDES DELGADO, J. (2013), Reflexiones en torno a la responsabilidad en tiempos de crisis, Universidad Pontificia Comillas, Madrid, pp. 23-42.

BAJO, A. (2013), La Responsabilidad Social en el ámbito de la crisis, Universidad Pontificia Comillas, Madrid, pp. 23-42.

BIRTH, G. (2008), "Communicating CSR: practices among Switzerland's top 300 companies", Corporate Communications: An International Journal 13, pp. 182-196.

BLÁZQUEZ, S. (2013), "La dimensión útil. Cuidar a los empleados, el entorno y los proveedores proporciona réditos económicos", El País, . Extra. Responsabilidad Social Corporativa. En el ADN de la empresa, pp. 8.

BOATRIGHT, J. (2010), "Business Ethics: Where Should the Focus Be?", Business Ethics Quarterly, pp. 711-712.

BOWEN, H. (1953), Social Responsibility of the Businessma,Harper $\mathcal{E}$ Row, New York.

BRAVO, R. y otros. (2012), "Corporate Social Responsibility as a Vehicle to Reveal the Corporate Identity: A Study Focused on the Websites of Spanish Financial Entities", Journal of Business Ethics, vol. 107, pp.129-146.

BRUCH, H. y WALTER, F. (2005), "The keys to rethinking corporate philanthropy", MIT Sloan Management Review, vol.47, pp. 48-56.

BURKE, L. y LOGSDON, J.M. (1996), "How Corporate Social Responsibility Pays Off”, Long Range Planning, vol. 29, núm. 4 , pp. 495-502.

CAMACHO, I. y otros (2013), Ética y Responsabilidad Empresarial, Desclée de Brower, Bilbao.

CANALS, L. (2010), "Rethinking the firm's mission and purpose", European Management Review, vol. 7 , pp. 195-204.

CARROLL, A. (1979), "A Three-Dimensional Conceptual Model of Corporate Performance", The Academy of Management Review, vol.4 , pp. 497-505.

CARROLL, A. y BUCHHOLTZ, A. (2009), Business $\mathcal{E}$ Society. Ethics and Stakeholder Managemen, South Western Cengage Learning,Mason, HO (USA).

CARROLL, A. y SABANA, K. (2010), "The Business Case for Corporate Social Responsibility: A Review of Concepts, Research and Practice", International Journal of Management Review , pp. 85-103. 
CARROLL, A. (1999), "Corporate Social Responsibilitry. Evolutiuon of Definitional Cosntruct”, Business Eु Society, vol. 38, núm.3 , pp. 268-295.

CARROLL, A. (1991), "The Pyramid of Corporate Social Responsibility: Toward the Moral Management of Organizational Stakeholders”, Business Horizons, pp. 39-48.

CERSE (2011), El papel de la RSE ante la crisis económica: su contribución a un nuevo modelo productivo, la competitividad y el desarrollo sostenible, http:// www.empleo.gob.es/es/sec_trabajo/autonomos/economia-soc/RespoSocEmpresas/consejo_rse/ Recuperado el 4 de Diciembre de 2013.

CLOUGH, S. B. (1990), Historia económica de Europa. El desarrollo económico de la civilización occidental, Omega, Barcelona.

COMÍN COMÍN, F. (2011), Historia económica mundial. De los orígenes a la actualidad. Alianza Editorial, Madrid.

COMISIÓN EUROPEA (2011), Comunicación de la Comisión Europea al Parlamento Europeo, al Consejo, al Comité Económico y Social Europeo y al Comité de las Regiones. Estrategia renovada de la UE para 2011 2014 sobre la responsabilidad social de las empresas: Recuperado el 4 de Diciembre de 2013, http://eur-lex.europa.eu/LexUriServ/LexUriServ. do?uri=COM:2011:0681:FIN:ES:PDF

COMMISSION ( 2011), Conclusions of The Financial Crisis Inquiry Report. Recuperado el 28 de Noviembre de 2013, de http:/fcic-static.law.stanford.edu/cdn_media/fcic-reports/fcic_final_report_full.pdf: http://fcic-static.law.stanford.edu/cdn_media/fcic-reports/fcic_final_report_full.pdf

COMMUNICATION WORLD. (2009), Is the Current Economic Environment Affecting the Way Organizations Approach Corporate Social Responsbility? Recuperado el 6 de Diciembre de 2013, de http:// www.questia.com/library/1G1-200340156/is-the-current-economicenvironment-affecting-the\#articleDetails: http://www.questia.com/ library/1G1-200340156/is-the-current-economic-environment-affecting-the\#articleDetails.

DAHLSRUD, A. (2008), "How corporate social responsibility is defined: an analysis of 37 definitions", Corporate Social Responsibility and Environmental Management, vol.15, pp. 1-13.

DORTIER, J-F. (2011), La gran historia del capitalismo, Globus, Madrid.

DRUCKER, P. F. (1984), "The new meaning of corporate social responsibility", California Management Review, 26 , pp. 53-63.

EAGLETON, T. (2011), Por qué Marx tenía razón, Península,Barcelona.

EFC, E. F. (2012), No es economía, es ideología, Deusto,Barcelona.

EKAIZER, E. (2012), Crónica de un atraco perfecto, Espasa,Madrid.

EKELUND, J. R. (2008), Historia de la Teoría Económica y su método, McGraw Hill, México. 
EUROPEAN COMMISSION (2009), Economic Crisis in Europe: Causes, Consequences and Responses, European Economy 7, Luxembourg.

FERNÁNDEZ-FEIJÓ, B. (2009), "Crisis and Corporate Social Responsibility: Threat or Opportunity?", International Journal of Economic Sciences and Applied Research, pp. 36-50.

FOMBRUM C. y SHANLEY, M (1990), "What's a name? reputation building and corporate strategy", Academy of Management Review, vol.33 , pp. 233-258.

FREDERICK, W. (1994), "From CSR1 to CSR2: The Maturing of Businessand-Society Thought", Business Ë Society, vol. 33 , núm. 2 , pp. 150-164.

FREDERICK, W. (1998), "Moving to CSR4: What to Pack for the Trip", Business $\mathcal{E}$ Society, vol. 37, núm 1, pp. 40-59.

FREDERICK, W. (1986), "Towards CSR3: Why Ethical Analysis is Indispensable and Unavoidable in Corporate Affaires" , California Management Review, vol. 28, núm. 2 , pp. 126-142.

FREEMAN, R. y PHILIPS, R(2002), "Stakeholder Theory: A Libertarian Defence”, Business Ethics Quarterly, vol. 12, núm 3, pp. 331-349.

FREEMAN, R. (1994), "The Politics of Stakeholder Theory: Some Future Directions", Business Ethics Quarterly, vol. 4, núm 4 , pp. 409-429.

FREEMAN R. y REED, D. (1983), "Stockholders and Stakeholders: A New Perspective on Corporate Governance”, California Management Review, vol. 25 , pp. 88-106.

FRIEDMAN, M. (1962), Capitalism anf Freedom, University of Chicago Press, Chicago.

FRIEDMAN, M. (1967), Ensayos sobre economía positiva, Gredos, Madrid.

FRIEDMAN, M. (1970), "The Social Responsibility of Business is to Increase its Profits”, New York Times Magazine, pp. 32-33,122,126.

FRIEDMAN, M. (1966),Capitalismo y Libertad, Rialp, Madrid.

GARCÍA VEGA, M. Á. (2013), "Responsables cotidianos", ElPaís, Extra Responsabilidad Social Corporativa: En el ADN de la empresa, pp. 2-3.

GARRIGA, E. y MELÉ, D. (2004),"Corporate Social Responsibility Theories: Mapping the Territory" Journal of Business Ethics, vol. 53, núm1/2 , pp. 51-71.

GIANNARAKIS, G. y THEOTOKAS, I. (2011), "The Effect of Financial Crisis in Corporate Social Responsibility Performance", International Journal of Marketing Studies, vol .3, núm. 1, pp. 2-10.

GIIANNARAKIS, G. y SARIANNIDIS, N (2012), "The Role of Corporate Social Responsibility in an Unstable Environment: An Examination of Trends 2008-2010", International Journal of Management, vol. 29, núm. 4, pp. 579-589.

GJOLBERG, M. (2009), "Measuring the immensurable? Constructing and index of CSR parctices and CSR performance in 20 countries", Scandinavian Journal of Management, pp.10-22. 
GÓMEZ-BEZARES, F. (1995), "Panorama de la teoría financiera”, Boletín de Estudios Económicos, núm. 156, pp.411-448.

GRAAFLAND, J. J. y VAN DE VEN, B. (2011), "The Credit Crisis and the Moral Responsibility of Professionals in Finance", Journal of Business Ethics, vol. 103 , pp. 605-619.

HAYEK, F. A. (2009), Camino de servidumbre, Alianza Editorial, Madrid.

IAMANDI, I.E. y otros. (2010), "Corporate Social Responsibility during the economic crisis, The case of the romanian companies", Annals of Faculty of Economics, University of Oradea, Faculty of Economics, vol 1(2) , pp. 963-969.

IGALENS, J. y GOND, J.P.(2005), "Measuring Corporate Social Performance in France: A Critical and Empirical Analysis of ARESE data”, Journal of Business Ethics, vol.56 (2) , pp. 131-148.

JENSEN, M. y MENCKLING, W. (1976), "Theory of the Firm: Managerial Behaviour, Agency Cost, and Capital Structure", Journal of Financial Economics, vol. 3, núm 4 , pp. 305-360.

JIN, K. R. (2013), "The Role of Corporate Value Clusters in Ethics, Social Responsibility, and Performance: A Study of Financial Professionals and Implications for the Financial Meltdown", Journal of Business Ethics, vol. 112 , pp. 15-24.

KARAUBRAHIMOGLU, Y. (2010), "Corporate Social Responsability in times of financial crisis", African Journal of Business Management, vol. 4 (4), pp. 382-389.

KELLY, M. y WHITE, W (2009), "From Corporate Responsibility to Corporate Design: Rethinking the Pourpose of the Corporation”, Journal of Corporate Citizenship, Spring, Issue 33, pp. 23-27.

KEMPER, A. y MARTIN, R.(2010), "After the fall: The global financial crisis as a test of corporate social responsibility theories”, European Management Review, vol. 7 , núm. 229-239.

KEYNES, J. M. (2004), Teoría general de la ocupación, el interés y el dinero,RBA, Barcelona.

KLONOSKI, R. (1991), "Foundational Considerations in the Corporate Social Responsibility Debate”, Business Horizons, vol. 34, núm. 4, pp. 9-18.

KOLK, A. (2010), "International Business, Corporate Social Responsibility and Sustainable Development", International Business Review, vol. 19, núm. 2 , pp.119-125.

KONDRATIEFF, N. D. (1944), Los grandes ciclos de la viad económica, en Urquidi V. L., Ensayos sobre el ciclo económico, Fondo de Cultura Económica, México, pp. 35-56.

KRAUS, P. y BRTITZELMAIER , B.(2012), “A literature review on corporate social responsibility: definitions, theories and recent empirical researches", International Journal of Management Cases, vol 14, Issue 4,pp. 282-296. 
KRUGMAN, P. (2012), ¡Acabad ya con esta crisis!, Crítica, Barcelona.

KURUCZ, E. B. (2008), "The business case for corporate social responsibility", en Crane A. A., The Oxford Handbook of Corporate Social Responsibility, Oxford University Press, Oxford, pp. 83-112.

LAPAVITSAS, C. (2009), El capitalismo financiarizado. Expansión y crisis. Maia Ediciones, Madrid.

LAWRENCE, A. y WEBER, M. (2011),Business and Society: Stakeholders, Ethics, Public Policy, $13^{a}$ ed. McGraw-Hill, New York.

LEE, M.D. P. (2008), "A review of the theories of corporate social responsibility: Its evolutionary path and the road ahead", International Journal of Management Reviews, vol. 10 (1) , pp. 53-73

MAIGNAN, I. (2000), "Measuring Corporate Citizenship in two Countries: The Case of the United States and France", Journal of Business Ethics, vol. 23 (3) , pp. 283-297.

MANUBENS, M. (2009), "Corporate Social Responsability in an Economic Crisis: An Opportunity for Renewal, Global Business and Organizational Excellence, pp. 50-60.

MARX, R. (2011), El capital. Un alegato a favor de la Humanidad, Planeta, Barcelona.

MASLOW A. H. (1975), Motivación y personalidad, Sagitario,Barcelona.

MATTEN, D. y CRANE, A. (2005),Corporate Citizenship: Towards an Extended Theoretical Conceptualization, Academy of Management Review, vol. 30, núm.1 , pp. 166-179.

MCGUIRE, J. (1963), Business and Society, McGraw-Hill. New York.

MCWILLIAMS , A. y SIEGEL, D. (2001), Corporate Social Responsibility: A Theory of the Firm Perspective, Academy of Management Review, vol. 26, núm. 1, pp. 117-127.

MELÉ, D. (2008), Corporate Social Responsibility Theories. En A. e. Crana, The Oxford Handbook of Corporate Social Responsibility, Oxford University Press, Oxford, pp. 47-82.

MELÉ, D. (2007), "Responsabilidad social de la empresa: una revisión crítica a las principales teorías", Ekonomiaz, vol. 65 , pp. 50-67.

MINISTERIOS DE ASUNTOS EXTERIORES Y COOPERACIÓN (2013), http://es.scribd.com/doc/187025965/2\%C2\%BA-Borrador-del-Plan-Nacionalde-Empresas-y-Derechos-Humanos-doc. Recuperado el 4 de Diciembre de 2013, de $2^{\circ}$ Borrador del Plan Nacional de Empresas y Derechos Humanos http://es.scribd.com/doc/187025965/2\%C2\%BA-Borrador-del-Plan-Nacional-de-Empresas-y-Derechos-Humanos-doc

MINTZBERG, H. (1983), "The case for corporate social responsibility", Journal of Business Strategy, vol. 4 , pp. 3-15.

MISES, L. (1995), La acción humana. Tratado de Economía, Unión Editorial, Madrid. 
MISES, L. (2011), La mentalidad anticapitalista, Unión Editorial, Madrid.

MITCHEL, R. B. (1997),"Towards a Theory of Stakeholder Identifications and Salience: Defining the Principle of Who and What Really Counts", Academy of Management Review, vol. 22, núm. 4, pp. 853-886.

MITCHELL, W. C. (1927), Business Cycles: The Problem and its Setting, National Bureau of Economic Research, New York.

MUÑOZ, P. A. ( 2013), La oficina sostenible pide paso, El País, Extra. responsabilidad Social Corporativa. En el ADN de la empresa, pp. 10.

NAPAL, G. (2013), Incorporating CSR and Sustainability in the Business Strategy. 3rd Annual International Conference on Business Strategy and Organizational Behaviour (BizStrategy 2013) , pp. 189-195.

NATALE, S. M. (2010), "Ethics in Strategic Thinking: Business Processes and the Global Market Collapse", Journal of Business Ethics, vol. 94 , pp. 309-316.

NAUDÉ, W. (2009), The Financial Crisis of 2008 and the Developing Countries, http://www.wider.unu.edu/publications/working-papers/discussionpapers/2009/en_GB/dp2009-01/Recuperado el 4 de Diciembre de 2013.

NJOROGE, J. (2009), Effects of the global financial crisis on corporate social responsibility in multinational companies in Kenya, http://www.ethicalquote. com/docs/Kenya-Crisis.pdf

Recuperado el 4 de Diciembre de 2013,

ORTEGA, A. (2012), ¿Qué nos ha pasado? El fallo de un país, Galaxia Gutenberg-Círculo de lectores, Barcelona.

PÉREZ-RUIZ, A. y RODRÍGUEZ, I.A. (2013), "Measuring CSR Image: Three Studies to Develop and to Validate a Reliable Measurement Tool", Journal of Business Ethics, vol. 118 , pp. 265-286.

(2012), "La imagen de la Responsabilidad Social Corporativa en un contexto de crisis económica: El caso del sector financiero en España", Universia Business Review , pp. 14-29.

PETRINI, R. (2010), Proceso a los economistas, Alianza, Madrid.

PORTER, M. E. (2006), "Strategy \& Society: the link between competitive advantage and corporate social responsibility", Harvard Business Review, vol 84 , pp. 78-92.

(2002), "The Competitive Advantage of Corporate Philanthropy", Harvard Business Review, vol. 80, pp. 56-69.

PRIVATO, S. N. (2008), "The impact of corporate social responsibility on consumer trust: the case of organic food", Business Ethics: A European Review, vol. 17, pp. 3-12.

SANTIAGO, E. ( 2013), "Regular las buenas intenciones", El País, Extra Responsabilidad Social Corporativa. En el ADN de la empresa, p. 4.

SETHI, S. (1975), "Dimensions of Corporate Social Performance: An Analytical Framework”, California Management Review, vol. 17, núm. 3, pp. 58-64. 
SIMÓN, A. ( 2013), "Míster RSC quiere hacer negocio", El País, Extra. Responsabilidad Social Corporativa. En el ADN de la empresa, p. 6.

SMITH, T. (2005), "Institutional and social investors find common ground", Journal of Investing, vol. 14 , pp. 57-65.

SUÁREZ SERRANO, M. E. (2013), "La responsabilidad social corporativa: un nuevo paradigma para las empresas", Encuentros Multidisciplinares, núm. 45, pp. 22-31.

SUCHMAN, M. (1995), "Managing legitimacy: strategic and institutional approaches", Academy of Management Journal, vol. 20 , pp. 571-610.

SUSTAINABILITY ASSET MANAGEMENT (2011), The Sustainability Year Book, SAM, New York.

TAPIADOR, T. (2013), "Comprar sin dejar huella!, El País. Extra. Responsabilidad Social Corporativa. En el ADN de la empresa, p. 14.

THOMÉ, F. (2009), Corporate responsibility in the age of irresponsibility;: A symbiotic relationship between CSR and the financial crisis? An IISD Comentary: http://www.iisd.org/pdf/2009/csr_financial_crisis.pdf Recuperado el 4 de Diciembre de 2013.

TORRES LÓPEZ, J. (2011), Contra la crisis, otra economía y otro modo de vivir, HOAC, Madrid.

TORTELLA, G. y NÚNEZ, C. E. (2010), Para comprender la crisis, Gadir Editorial, Madrid.

TURKER, D. (2009), "Measuring Corporate Social Responsibility: A Scale Development Study", Journal of Business Ethics, vol. 85 (4) , pp. 411-427.

UNITED NATIONS HUMAN RIGHTS, (2011), Guiding Principles on Business and Human Rights. Implementing the United Nations "Protect, Respect and Remedy" Framework.

http://www.ohchr.org/Documents/Publications/GuidingPrinciplesBusinessHR_EN.pdf. Recuperado el 4 de Diciembre de 2013.

VALOR, C. (2008), "Can consumers buy responsibly' Analysis and solutions for market failures", Journal of Consumer Policy, vol. 31, pp. 315-326.

VOLOSEVICI, D. (2013), "Corporations-between Governance and Social Responsibility: the European Commission Approach”, Economic InsightsTrends and Challenges, pp. 124-135.

WADDOCK, S. y SMITH, N (2000), "Relationship: The Real Challenge of Corporate Global Citizenship”,Business $\mathcal{E}$ Society Review, vol. 105, núm. 1 , pp. 47-62.

WARTICK, S. y COCHRAN, P.(1985), "The Evolution of Corporate Social Performance Model”,Academy of Mangement Review, vol. 10, núm. 4, pp. 758-769.

WHEELER, C. B. (2003), "Focusing on value: reconciling corporate social responsibility, sustainability and a stakeholder approach in a network world", Journal of General Management, vol. 28 (3), pp. 1-28. 
WILLIAMSON, D. y otros (2006), "Drivers of environmental behaviour in manufacturing SMEs and the implications for CSR", Journal of Business Ethics, vol. 67, pp. 317-330.

WINDSOR, D. (2006), "Corporate Social Responsibility: Three Key Approaches", Journal of Management Studies,vol. 43, núm. 1 , pp. 93-114.

WOOD, D. (2002), "Business Citizhenship: From Individuals to Organizations", Business Ethics Quarterly, Ruffin Series, núm. 3 ,pp. 59-94. (1991), "Corporate Social Performance Revisited", Academy of Management Review, vol. 16, núm. 4 , pp. 691-718.

YELKIKALAN, N. y KÖSE, C . (2012), "The effects of the financial crisis on the corporate social responsibility", International Journal of Business ans Social Science, vol. 3, núm. 3, pp. 292-300.

ZAMAGNI, S. (2012), "El fundamento ético y la crítica a la responsabilidad social de la empresa", en Zamagni, S., Por una economía del bien común, Editorial Ciudad Nueva, Madrid, pp. 175-203 